\title{
AVVERTENZE PER AUTORI E COLLABORATORI
}

Gli autori sono pregati di osservare le seguenti norme redazionali:

1. Gli articoli devono essere inviati in tre copie, accompagnate da un riassunto di circa 15-20 righe in inglese (anche il titolo deve essere tradotto). I dattiloscritti non vengono restituiti agli autori. Nome e indirizzo dell'autore (o degli autori) devono essere riportati separatamente in un foglio allegato, unitamente ad una breve nota bio-bibliografica (massimo 5-6 righe dattiloscritte). Il materiale deve essere inviato al seguente indirizzo: Rivista Italiana di Scienza Politica c/o Dipartimento di scienze storiche, giuridiche, politiche e sociali, piazza S. Francesco 7, 53100 Siena.

2. I dattiloscritti devono essere battuti a spazio 2 (circa 2.000 battute per cartella), su una sola facciata con ampi margini. Figure, tabelle e grafici devono essere allegati a parte. Nel testo deve invece risultare ben chiaro il punto dove si desidera vengano inseriti. Note e didascalie di figure e di grafici devono a loro volta essere riportate su fogli a parte. La redazione provvederà a richiedere agli autori il testo su dischetto degli articoli accettati.

3. In generale, i riferimenti bibliografici vanno inseriti direttamente nel testo, riportando soltanto il cognome dell'autore, l'anno di pubblicazione del lavoro e - se necessario - la pagina o le pagine cui ci si riferisce. Esempi: Easton (1953) o (Easton 1953) oppure (Easton 1953, 62). Nel caso in cui gli autori siano più di due si può usare l'abbreviazione et al. Quando più opere dello stesso autore o degli stessi autori sono state pubblicate nello stesso anno, si usino le indicazioni a, b, c. Ad esempio (Rose 1974a) e Rose (1974b). La stessa procedura deve essere usata per le indicazioni bibliografiche contenute nelle note a piè di pagina. 
4. Alla fine del testo i riferimenti bibliografici vanno elencati in ordine alfabetico secondo il cognome degli autori e, per ciascun autore, nell'ordine cronologico di pubblicazione delle opere. Nel caso di lavori di più autori, devono essere riportati i cognomi di tutti. Nel caso di un lavoro curato da un autore, va riportato il nome del curatore seguito dalla dizione (a cura di), anche per le edizioni in lingua straniera. Quando si cita un'edizione originale in lingua straniera va segnalata l'eventuale traduzione italiana. I riferimenti bibliografici vanno redatti secondo regole desumibili dai seguenti esempi:

Almond, G.A. e B.G. Powell (1966), Comparative Politics: A Developmental Approch, Boston, Little, Brown \& Co.; trad. it. Politica comparata, Bologna, Il Mulino, 1970.

Daalder, H. e P. Mair (a cura di) (1983), Western European Party Systems. Continuity and Change, London, Sage.

Hirschman, A.O. (1987), Il concetto di interesse: dall'eufemismo alla tautologia, in «Rivista Italiana di Scienza Politica», XVII, n. 1, pp. 3-22.

Lipset, S.M. (1981a), The Industrial Proletariat in Comparative Perspective, in J.K. Triska e C. Gati (a cura di), Blue Collar Workers in Eastern Europe, London, Allen \& Unwin, pp. 129.

- (1981b), The Revolt Against Modernity, in P. Torsvik (a cura di), Mobilization, Center-Periphery Structures and $\mathrm{Na}$ tion Building, Bergen, Universitäsvorlaget, pp. 451-500.

5. Le bozze da correggere vanno restituite entro i termini richiesti dalle esigenze della redazione; trascorso tale periodo di tempo si procederà alla correzione in redazione; le modifiche devono essere ridotte al minimo (in linea di massima le sole modifiche accettabili sono le correzioni degli errori di stampa); il costo di eventuali correzioni d'autore dovrà essere addebitato all'autore stesso.

6. L'editore riserva gratuitamente agli autori trenta estratti e non fornisce estratti anticipati o in soprannumero.

7. I libri per recensione dovranno essere inviati al Prof. Leonardo Morlino, via S. Caterina di Alessandria 3, 50129 Firenze.

Responsabile: Leonardo Morlino - Stampa: Grafiche Galeati - Imola, con i tipi della Centro Immagine P. (Capannori - LU). Registrato al n. 4110 presso la Cancelleria del Tribunale Civile Penale di Bologna, il 14-12-1970 - Copyright (c) 1994 by Società editrice il Mulino, Bologna. 